\title{
Incidence of Scleritis and Episcleritis: Results From the Pacific Ocular Inflammation Study
}

Gelareh Homayounfar, Natalie Nardone, Durga S. Borkar, Vivien M. Tham, Travis C. Porco, Wayne T.A. Enanoria, John V. Parker, Aleli C. Vinoya, Aileen Uchida, and Nisha R. Acharya F.I. Proctor Foundation (G.H., N.N., D.S.B., T.C.P., W.T.A.E., N.R.A.), and the Departments of Epidemiology and Biostatistics (T.C.P., N.R.A.) and Ophthalmology (T.C.P., N.R.A.), University of California, San Francisco, California; and the Department of Ophthalmology (V.M.T.) and Center for Health Research (J.V.P., A.C.V., A.U.), Kaiser Permanente Hawaii, Honolulu, Hawaii

\begin{abstract}
Purpose-To ascertain the incidence of scleritis and episcleritis in a Hawaiian population and describe variations by age, sex, and race.

Design-Retrospective, population-based cohort study.

Methods-All electronic medical records for enrollees in Kaiser Permanente Hawaii ( $\mathrm{n}=217$ 061) from January 1, 2006 to December 31, 2007 were searched for International Classification of Diseases, 9th Edition (ICD-9) codes associated with ocular inflammation. Chart review was conducted to verify a clinical diagnosis of scleritis or episcleritis. Confirmed cases were used to calculate incidence rates per 100000 person-years. Ninety-five percent confidence intervals (CI) were calculated for each incidence rate, including age-, sex-, and race-specific rates, using biascorrected Poisson regression. To assess for confounding, a multivariate analysis adjusting for age, sex, and race was also performed.
\end{abstract}

Results-Of 217061 eligible patients, 17 incident scleritis cases and 93 incident episcleritis cases were confirmed. The overall incidence rates of scleritis and episcleritis were 4.1 (95\% CI: 2.6-6.6) and 21.7 (95\% CI: 17.7-26.5) cases per 100000 person-years, respectively. Women were overrepresented among scleritis patients $(P=.049)$. Pacific Islanders were the most underrepresented racial group among cases of scleritis and episcleritis $(P=.006, P=.001)$. Blacks had the highest incidence of scleritis $(P=.004)$.

Conclusions-These results provide a population-based estimate of the incidence of scleritis and episcleritis in a diverse population and highlight differences in patients' demographic characteristics. Differences in incidence by sex and race raise questions about genetic and environmental influences on the development of these conditions.

\section{Cleritis and Episcleritis are Ocular Inflamma}

Story diseases with different implications for visual outcomes and systemic disease risk. Episcleritis has been classically described as having a self-limited course and posing no threat to vision, ${ }^{1,2}$ whereas scleritis may result in ocular complications, be more commonly associated with systemic diseases and increased mortality, and require systemic immunosuppressive therapy. ${ }^{1-14}$ To date, studies of these diseases have been limited to

\footnotetext{
(C) 2013 by Elsevier Inc.All rights reserved. Inquiries to Nisha R. Acharya, Room S309, 513 Parnassus Ave, UCSF, San Francisco, CA 94143-0412; nisha.acharya @ucsf.edu. Supplemental Material available at AJO.com

All Authors have Completed and Submitted the ICMJE form for Disclosure of potential Conflicts of Interest and none were reported.
} 
retrospective case series from tertiary eye care centers, which are susceptible to referral bias. One study, published in 1974, found that patients with scleritis made up $0.08 \%$ of referrals to a hospital ophthalmology department..$^{15}$ Two more recent studies, both from tertiary eye care centers, found that $2.6 \%-8.7 \%$ of referrals to ocular inflammatory disease specialists suffered from scleritis and $1.4 \%$ from episcleritis. ${ }^{3,16}$ Given the clinical importance of scleritis and episcleritis, the limitations of existing incidence data, and the absence of population-based reports, there is a need for a population-based study characterizing their epidemiology.

There are several advantages to considering Kaiser Permanente Hawaii as a source of data for a population-based study. Kaiser serves approximately $16 \%$ of Hawaii's total population, with 18 clinics throughout the state. ${ }^{17,18}$ Furthermore, the population served is racially diverse and includes all age groups. Additionally, patient encounters are documented in a readily accessible electronic medical records system.

This epidemiologic study seeks to ascertain the incidence of scleritis and episcleritis among Kaiser Permanente Hawaii members and to describe demographic variations in incidence by age, sex, and race.

\section{Methods}

Institutional Review Board (IRB)/Ethics Committee approval was prospectively obtained at Kaiser Permanente Hawaii and the University of California, San Francisco for all aspects of this study involving retrospective review of patient data. All work was Health Insurance Portability and Accountability Act compliant. Patient encounters documented between January 1, 2006 and December 31, 2007 in the electronic medical records of Kaiser Permanente Hawaii enrollees $(n=217061)$ were retrospectively queried using diagnosis codes from the International Classification of Diseases, 9th edition (ICD-9), listed in the Supplemental Table (available at AJO.com), corresponding to ocular inflammatory conditions. Since the larger Pacific Ocular Inflammation Study is interested in the epidemiology of ocular inflammatory diseases, including uveitis, herpetic eye disease, scleritis, and episcleritis, the ICD-9 codes initially chosen were broad. Subsequently, a uveitis and cornea fellowship-trained ophthalmologist verified all patient diagnoses based on documented clinical exams. The main outcome measure of this study was a new clinical diagnosis of scleritis or episcleritis during the study period, and only confirmed cases were included in incidence calculations. Demographic data, including age, sex, and self-reported race, were collected and compared between incident cases and the general Kaiser Permanente Hawaii membership using Fisher exact test. Overall incidence rates and incidence rates across demographic groups were calculated.

Incidence rates were calculated per 100000 person-years by dividing the confirmed incident cases by the total person-years of follow-up and multiplying by 100000 . Given the constant fluctuation in membership of the Kaiser Permanente Hawaii health system, we used quarterly population data to calculate the total number of person-years of follow-up. Incidence rates were reported for the overall study period (January 1, 2006-December 31, 2007) and also within each year to evaluate the consistency of the rates. We separately calculated population-based rates for scleritis and for episcleritis, and stratified them by age and sex. Age was stratified into the following groups: 0-14, 15-24, 25-44, 45-64, and 65+ years of age, based on patient age at the middle of the study period, January 1,2007 . When calculating age- and sex-specific incidence rates, the total number of person-years was adjusted to reflect quarterly membership data within each group. Since race data were available only for a portion of incident cases and Kaiser Permanente Hawaii members, 
incidence analyses by race reflect the demographics of the subset of patients who selfreported their race.

We calculated $95 \%$ confidence intervals (CI) for each incidence rate and compared age-, sex-, and race-specific incidence rates using bias-corrected Poisson regression. ${ }^{19,20}$ This method is designed to yield small positive incidence estimates even when zero counts are observed for a given number of person-years of observation. ${ }^{20}$ Univariate and multivariate analyses were conducted using age category, sex, and racial classification. Relative rates were estimated by using bias-corrected Poisson regression, with the logarithm of the number of person-years at risk as the offset. Approximate confidence intervals were constructed using the estimated standard errors. Likelihood ratio tests were used to assess the statistical significance of race. ${ }^{21}$ A P value less than .05 was considered statistically significant. STATA 11.0 (StataCorp, College Station, Texas, USA) and R statistical software (The R Foundation for Statistical Computing, Vienna, Austria) were used for performing all analyses.

\section{Results}

Kaiser Permanente Hawaii Had a total Mid-Study period population of 217061 people, which corresponds to a person-time estimate of 433794.2 person-years for the study period. From this population, 873 patient records were identified on the basis of ICD-9 diagnosis code search (Supplemental Table, available at AJO.com). Based on individual review of these medical records, there were 17 confirmed scleritis cases and 93 confirmed episcleritis cases (Figure). Notable reasons for exclusion include diagnoses of herpes simplex virus/ herpes zoster virus (HSV/HZV) eye disease not involving the sclera or episclera, uveitis, invalid diagnoses, traumatic or postsurgical inflammation, contact lens-related keratitis, nonincident cases of scleritis or episcleritis, pinguecula, and pterygia.

Demographic characteristics of scleritis and episcleritis cases and the general Kaiser Permanente Hawaii population were compared (Table 1). Statistically significant differences by sex and race were noted. Women were over-represented among scleritis patients $(P=$. 0498). Additionally, Pacific Islanders were the most underrepresented racial group among cases of scleritis and episcleritis compared to the general Kaiser Permanente Hawaii membership $(P=.006, \mathrm{P}=.001)$. Blacks made up a significantly higher proportion of scleritis cases compared to the Kaiser Permanente Hawaii population $(P=.03)$.

The overall incidence rates of scleritis and episcleritis were 4.1 (95\% CI: 2.6-6.6) and 21.7 (95\% CI: 17.7-26.5) per 100000 person-years, respectively. Separate incidence calculations for each year in the study period yielded similar rates (not reported).

Incidence rates by age and sex subgroup are also reported in Table 2 . The incidence rate of scleritis increases with age; however, variations in incidence by age category were not statistically significant. Additionally, we found that only adults had scleritis, whereas episcleritis affected all age groups. The median age at diagnosiswas 56 years for scleritis (interquartile range [IQR]: 42-60) and 45 years for episcleritis (IQR: 32-54). Of scleritis cases, $76.5 \%$ (13/17) were female; of episcleritis cases, 60.2\% (56/93) were female. Incidence rates varied by sex. For scleritis, female subjects had an incidence of 6.4 (95\% CI: 3.8-10.8) per 100000 person-years and male subjects had an incidence of 2.3 (95\% CI: 1.0-5.6) per 100000 person-years. For episcleritis, female subjects had an incidence of 26.0 (95\% CI: 20.1-33.7) per 100000 person-years and male subjects had an incidence of 17.7 (95\% CI: 12.9-24.3) per 100000 person-years. 
Estimations of incidence were also made by racial subgroup (Table 3). Notably, Pacific Islanders had the lowest incidence of scleritis, 1.6 (95\% CI: 0.2-11.3) cases per 100000 person-years, and the lowest incidence of episcleritis, 19.1 (95\% CI: 10.8-33.6) cases per 100000 person-years. Blacks had the highest incidence of scleritis, 83.9 (95\% CI:

27.1-260.2) cases per 100000 person-years. Whites had the highest incidence of episcleritis, 48.6 (95\% CI: 34.0-69.5) cases per 100000 person-years.

The results of a multivariate model adjusting for age, sex, and race were as follows. Adjusting for sex and race, differences in the incidence of scleritis and episcleritis by age were not statistically significant $(P=.55, \mathrm{P}=.90$, respectively). The relative rate of scleritis in male subjects was 0.4 (95\% CI: 0.1-1.2) compared to female $(P=.09)$. Similarly, the relative rate of episcleritis in male subjects was 0.6 (95\% CI: 0.4-1.0) compared to female ( $P$ $=.07)$. Overall, race was a significant predictor of scleritis $(\mathrm{P}=.03)$ in a model adjusting for sex and age (likelihood ratio test with 4 degrees of freedom). However, for episcleritis, race was not a significant predictor ( $P=.16$, likelihood ratio test with 4 degrees of freedom). Blacks had a 9.3 times higher relative rate (95\% CI: 2.0-42.4) of having scleritis than whites $(P=.004)$. Compared to whites, Pacific Islanders' relative rate of scleritis was 0.1 (95\% CI: $0.01-1.7, P=.11$ ), which was not statistically significant, and their relative rate of episcleritis was 0.4 (95\% CI: $0.2-0.8, P=.01$ ). Compared to whites, the relative rate of episcleritis in Asians was 0.6 (95\% CI: 0.4-1.1, $P=.06$ ).

\section{Discussion}

Past Studies of Scleritis And Episcleritis from tertiary care centers and specialty practices have been limited by referral bias. In this epidemiologic study, we ascertained populationbased incidence rates of scleritis and episcleritis and assessed variations across demographic groups.

Overall incidence rates of 4.1 per 100000 person-years for scleritis and 21.7 per 100000 person-years for episcleritis were found in this population. Prior studies from tertiary care centers and ocular inflammatory disease specialty practices do not offer insight into incidence in a general population. Our results suggest that these conditions are overrepresented in such practices, as expected, and that episcleritis is more common than scleritis in a general population.

Our finding of a female predominance in scleritis and episcleritis is consistent with retrospective case series reporting that $60 \%-74 \%$ of patients with scleritis $1,3-5,7,8,10,11,14,22,23$ and $45 \%-70 \%$ of patients with episcleritis are women. ${ }^{1,3-6}$ This is also consistent with sex differences found in many systemic autoimmune and inflammatory conditions, such as multiple sclerosis (MS), rheumatoid arthritis, and systemic lupus erythematosus. ${ }^{24-26}$ With respect to age, we found that the median age at diagnosis was 56 years for scleritis and 45 years for episcleritis. This was consistent with reported findings that patients with scleritis most commonly present between the ages of 47 and $59.5,{ }^{3-5,7,8,10,11,14,22,23}$ and patients with episcleritis most commonly present between the ages of 43 and $47 .^{3,5,6}$

Limited data are available regarding racial differences in the risk of scleritis and episcleritis. One retrospective chart review from a tertiary care center described the racial composition of their referral population as either white or nonwhite and found that patients with scleritis are $79.4 \%$ white and $20.6 \%$ nonwhite, and patients with episcleritis are $83.8 \%$ white and $16.2 \%$ nonwhite $(P=.70){ }^{5}$ Additionally, a retrospective review of infectious scleritis cases seen at Bascom Palmer Eye Institute found that the racial distribution of cases was reflective of the Miami population. ${ }^{27}$ Although Pacific Islanders were well represented among Kaiser 
Permanente Hawaii members, had the second-highest person-time at risk, and had the lowest incidence of scleritis and episcleritis, the lower relative rate did not reach statistical significance with respect to scleritis. In contrast, though there are relatively few blacks in the Kaiser Permanente Hawaii population, they suffered the highest incidence of scleritis, which was a statistically significant finding. Furthermore, though blacks had the highest incidence rate of scleritis, they experienced no episodes of episcleritis. This apparent discrepancy is likely attributable to the small sample size of this racial group in our population.

Other epidemiologic studies have shown racial differences in disease incidence of uveitis, as well as other autoimmune diseases, that are consistent with our findings. Blacks have a higher incidence of sarcoidosis, ${ }^{28}$ systemic lupus erythematosus, ${ }^{29}$ polymyositis, ${ }^{30}$ and systemic sclerosis. ${ }^{31}$ Whites more commonly develop giant cell arteritis, ${ }^{32}$ type I diabetes mellitus, ${ }^{33}$ and granulomatosis with polyangiitis. ${ }^{34}$ A population-based study of uveitis showed that Pacific Islanders had a lower prevalence rate than non-Pacific Islanders, while whites had a higher prevalence rate than nonwhites ${ }^{35}$ Additionally, Asians/Pacific Islanders have lower rates of MS, ${ }^{36}$ type I diabetes mellitus, ${ }^{33}$ and systemic lupus erythematosus ${ }^{29}$ than others. A study of MS mortality showed that whites had the highest age-adjusted mortality rates and Asians/Pacific Islanders had the lowest. ${ }^{37}$ These results highlight the possibility of genetic or environmental influences on the development of ocular inflammatory conditions, as well as other conditions influenced by autoimmunity. Future studies are needed to elucidate the genetic, epigenetic, geographic, and socioeconomic factors that may contribute to the development of scleritis and episcleritis.

A few limitations of the study must be considered. It is possible that cases were missed because of patients seeking out-of-network care or miscoding of diagnoses. However, outof-network care is unlikely given Hawaii's geographic separation from the mainland United States and the fact that less than 5\% of Kaiser Permanente Hawaii members have alternate insurance. Additionally, the possibility of miscoding was minimized by an initially broad search of ICD-9 codes corresponding to ocular inflammatory conditions and subsequent chart review, which was needed to distinguish scleritis and episcleritis, entities with overlapping ICD-9 codes. Data on infectious vs noninfectious causes and the location of inflammation in scleritis and episcleritis were not collected during the chart review. Additionally, since episcleritis may have minimal symptoms and resolve spontaneously, patients may not have sought care, leading to underestimation of the incidence. Other potential causes of incorrect case ascertainment include misclassification and misdiagnosis. In a retrospective study, it is not possible to verify the physical examination or diagnostic criteria used. We trusted the treating physician's documentation of a diagnosis of scleritis or episcleritis, with documentation of corroborating examination findings and without past ocular history or examination findings suggestive of other diagnoses (eg, contact lensrelated keratitis, pinguecula, etc). It was our general impression from the records that questions regarding prior episodes of inflammation were asked as part of the clinical history, and there were 25 patients with scleritis or episcleritis whose diagnoses occurred outside of the study period and who were therefore excluded from incidence calculations. If prior episodes of scleritis or episcleritis were missed in the medical records, some cases of recurrent scleritis or episcleritis may have been misclassified as new cases. If so, this would result in overestimation of the incidence rates. Misclassification of scleritis as episcleritis or vice versa also could have affected estimates if misclassification was unbalanced. These are unavoidable limitations of the retrospective methodology.

Another potential limitation of our study is in the statistical analysis, as multiple comparisons could increase the chance of spurious associations. However, we tried to limit comparisons to the number necessary to answer our study questions. Furthermore, since race data were not available for all patients within Kaiser Permanente Hawaii, our estimates of 
incidence by race are based on reported data. These estimates may be biased if reported race data are not missing at random, but are instead underreported in specific groups.

As with other epidemiologic studies, generalizability of our results to other populations is a concern. Although nearly $20 \%$ of the Hawaiian population is enrolled in Kaiser Permanente Hawaii, these patients may not be representative of the entire US population. There is a higher proportion of Asians and Pacific Islanders and a lower proportion of whites, blacks, and Native Americans in the Kaiser Permanente Hawaii membership than in the general US population. Despite this, our study yields useful population-based estimates for a diverse group of patients and ascertains differences among racial groups that might not be possible in other populations.

In summary, our study ascertains incidence rates of scleritis and episcleritis of 4.1 and 21.7 cases per 100000 person-years, respectively, in a diverse Hawaiian population. Our results confirm a female predominance that has been observed in previous studies from tertiary care centers, and show that race is an important predictor of scleritis. These differences in demographic characteristics raise questions about genetic and environmental influences on the development and progression of these conditions.

\section{Supplementary Material}

Refer to Web version on PubMed Central for supplementary material.

\section{Acknowledgments}

This work was supported by a grant from the Doris Duke Charitable Foundation to UCSF to fund Clinical Research Fellow Ms Homayounfar. Dr Acharya was supported by National Eye Institute grant K23EY017897 and is now supported by NEI grant U10 EY021125-01 and a Research to Prevent Blindness Career Development Award. Dr Porco and Dr Enanoria were supported for statistical consulting by That Man May See Foundation, UCSF. The UCSF Department of Ophthalmology is supported by National Eye Institute grant EY06190 and an unrestricted grant from the Research to Prevent Blindness Foundation. The sponsors or funding organizations had no role in the design or conduct of this research. Contributions of authors: design and conduct of the study (N.R.A., G.H., V.M.T.); collection, management, analysis, and interpretation of the data (N.R.A., G.H., N.N., D.S.B., V.M.T., J.V.P., A.C.V., A.U., T.C.P., W.T.A.E.); and preparation, review, or approval of the manuscript (N.R.A., G.H., N.N., D.S.B., V.M.T., J.V.P., A.C.V., A.U., T.C.P., W.T.A.E.).

\section{References}

1. Watson PG, Hayreh SS. Scleritis and episcleritis. Br J Ophthalmol. 1976; 60(3):163-191. [PubMed: 1268179]

2. Okhravi N, Odufuwa B, McCluskey P, et al. Scleritis. Surv Ophthalmol. 2005; 50(4):351-363. [PubMed: 15967190]

3. Sainz de la Maza M, Molina N, Gonzalez-Gonzalez LA, et al. Clinical characteristics of a large cohort of patients with scleritis and episcleritis. Ophthalmology. 2012; 119(1):43-50. [PubMed: 21963265]

4. Lyne AJ, Pitkeathley DA. Episcleritis and scleritis. Association with connective tissue disease Arch Ophthalmol. 1968; 80(2):171-176.

5. Jabs DA, Mudun A, Dunn JP, et al. Episcleritis and scleritis: clinical features and treatment results. Am J Ophthalmol. 2000; 130(4):469-476. [PubMed: 11024419]

6. Akpek EK, Uy HS, Christen W, et al. Severity of episcleritis and systemic disease association. Ophthalmology. 1999; 106(4):729-731. [PubMed: 10201593]

7. Raiji VR, Palestine AG, Parver DL. Scleritis and systemic disease association in a community-based referral practice. Am J Ophthalmol. 2009; 148(6):946-950. [PubMed: 19837380]

8. Akpek EK, Thorne JE, Qazi FA, et al. Evaluation of patients with scleritis for systemic disease. Ophthalmology. 2004; 111(3):501-506. [PubMed: 15019326] 
9. Sainz de la Maza M, Jabbur NS, Foster CS. Severity of scleritis and episcleritis. Ophthalmology. 1994; 101(2):389-396. [PubMed: 8115160]

10. Sainz de la Maza M, Molina N, Gonzalez-Gonzalez LA, et al. Scleritis therapy. Ophthalmology. 2012; 119(1):51-58. [PubMed: 22015381]

11. Erkanli L, Akova YA, Guney-Tefekli E, et al. Clinical features, prognosis, and treatment results of patients with scleritis from 2 tertiary eye care centers in Turkey. Cornea. 2010; 29(1):26-33. [PubMed: 19907295]

12. Foster CS, Forstot SL, Wilson LA. Mortality rate in rheumatoid arthritis patients developing necrotizing scleritis or peripheral ulcerative keratitis. Effects of systemic immunosuppression. Ophthalmology. 1984; 91(10):1253-1263. [PubMed: 6514289]

13. Akova YA, Jabbur NS, Foster CS. Ocular presentation of polyarteritis nodosa. Clinical course and management with steroid and cytotoxic therapy. Ophthalmology. 1993; 100(12):1775-1781. [PubMed: 7903120]

14. McCluskey PJ, Watson PG, Lightman S, et al. Posterior scleritis: clinical features, systemic associations, and outcome in a large series of patients. Ophthalmology. 1999; 106(12):2380-2386. [PubMed: 10599675]

15. Williamson J. Incidence of eye disease in cases of connective tissue disease. Trans Ophthalmol Soc U K. 1974; 94(3):742-752. [PubMed: 4534021]

16. Foster, CS.; Sainz de la Maza, M. The Sclera. New York: Springer-Verlag; 1994.

17. US Census Bureau. [Accessed September 13, 2012] Hawaii Quick Facts. Available at http:// quickfacts.census.gov/qfd/states/15000.html

18. Kaiser Permanente Hawaii. [Accessed September 13, 2012] Our Business Structure: Hawaii. Available at http://www.kaiserpermanentejobs.org/hawaii.aspx

19. Cameron, AC.; Trivedi, PK. Regression Analysis of Count Data. Cambridge: Cambridge University Press; 1998.

20. Firth D. Bias reduction of maximum-likelihood-estimates. Biometrika. 1993; 80(1):27-38.

21. Heinze, G. Technical Report 10/1999. Vienna: Section for Clinical Biometrics, CeMSIIS, Medical University of Vienna; 1999. The application of Firth's procedure to Cox and logistic regression.

22. Lachmann SM, Hazleman BL, Watson PG. Scleritis and associated disease. Br Med J. 1978; 1(6105):88-90. [PubMed: 620214]

23. Ahn SJ, Oh JY, Kim MK, et al. Clinical features, predisposing factors, and treatment outcomes of scleritis in the Korean population. Korean J Ophthalmol. 2010; 24(6):331-335. [PubMed: 21165230]

24. Oliver JE, Silman AJ. Why are women predisposed to autoimmune rheumatic diseases? Arthritis Res Ther. 2009; 11(5):252. [PubMed: 19863777]

25. Greer JM, McCombe PA. Role of gender in multiple sclerosis: clinical effects and potential molecular mechanisms. J Neuroimmunol. 2011; 234(1-2):7-18. [PubMed: 21474189]

26. Jimenez S, Cervera R, Font J, et al. The epidemiology of systemic lupus erythematosus. Clin Rev Allergy Immunol. 2003; 25(1):3-12. [PubMed: 12794256]

27. Hodson KL, Galor A, Karp CL, et al. Epidemiology and visual outcomes in patients with infectious scleritis. Cornea. 2013; 32(4):466-472. [PubMed: 22902495]

28. Rybicki BA, Major M, Popovich J Jr, et al. Racial differences in sarcoidosis incidence: a 5-year study in a health maintenance organization. Am J Epidemiol. 1997; 145(3):234-241. [PubMed: 9012596]

29. Chakravarty EF, Bush TM, Manzi S, et al. Prevalence of adult systemic lupus erythematosus in California and Pennsylvania in 2000: estimates obtained using hospitalization data. Arthritis Rheum. 2007; 56(6):2092-2094. [PubMed: 17530651]

30. Medsger TA Jr, Dawson WN Jr, Masi AT. The epidemiology of polymyositis. Am J Med. 1970; 48(6):715-723. [PubMed: 5420557]

31. Mayes MD, Lacey JV Jr, Beebe-Dimmer J, et al. Prevalence, incidence, survival, and disease characteristics of systemic sclerosis in a large US population. Arthritis Rheum. 2003; 48(8):22462255. [PubMed: 12905479] 
32. Pereira LS, Yoon MK, Hwang TN, et al. Giant cell arteritis in Asians: a comparative study. Br J Ophthalmol. 2011; 95(2):214-216. [PubMed: 20584707]

33. Dabelea D, Bell RA, D'Agostino RB Jr, et al. Incidence of diabetes in youth in the United States. JAMA. 2007; 297(24):2716-2724. [PubMed: 17595272]

34. Mahr A, Guillevin L, Poissonnet M, et al. Prevalences of polyarteritis nodosa, microscopic polyangiitis, Wegener's granulomatosis, and Churg-Strauss syndrome in a French urban multiethnic population in 2000: a capture-recapture estimate. Arthritis Rheum. 2004; 51(1):92-99. [PubMed: 14872461]

35. Acharya NR, Tham V, Esterberg E, et al. Incidence and prevalence of uveitis: results from the Pacific Ocular Inflammation Study. JAMA Ophthalmology. forthcoming.

36. Wallin MT, Culpepper WJ, Coffman P, et al. The Gulf War era multiple sclerosis cohort: age and incidence rates by race, sex and service. Brain. 2012; 135(Pt 6):1778-1785. [PubMed: 22628389]

37. Redelings MD, McCoy L, Sorvillo F. Multiple sclerosis mortality and patterns of comorbidity in the United States from 1990 to 2001. Neuroepidemiology. 2006; 26(2):102-107. [PubMed: 16374035]

\section{Biographies}

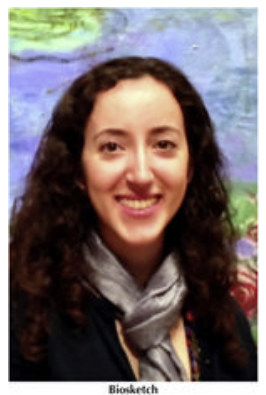

Gelareh Homayounfar, BS, is a fourth year medical student at Harvard University and a Doris Duke Clinical Research Fellow at the F.I. Proctor Foundation at UCSF, where she is studying ocular inflammatory diseases under the mentorship of Dr Nisha Acharya. She obtained a Bachelor of Science degree from Duke University, where she majored in Biology and Philosophy, graduating Phi Beta Kappa and Magna cum laude. She plans a career in academic ophthalmology.

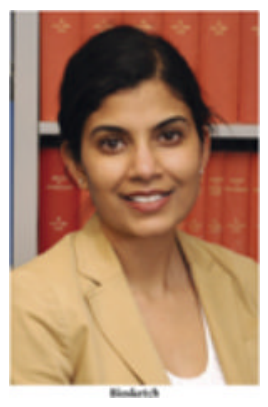

Nisha R. Acharya, MD, MS, is Director of the Uveitis Service at the F.I. Proctor Foundation and Associate Professor in the Departments of Ophthalmology and Epidemiology and Biostatistics at the University of California, San Francisco. Dr Acharya's research interests include designing and implementing clinical trials and epidemiologic studies in the field of ocular infection and inflammation. 


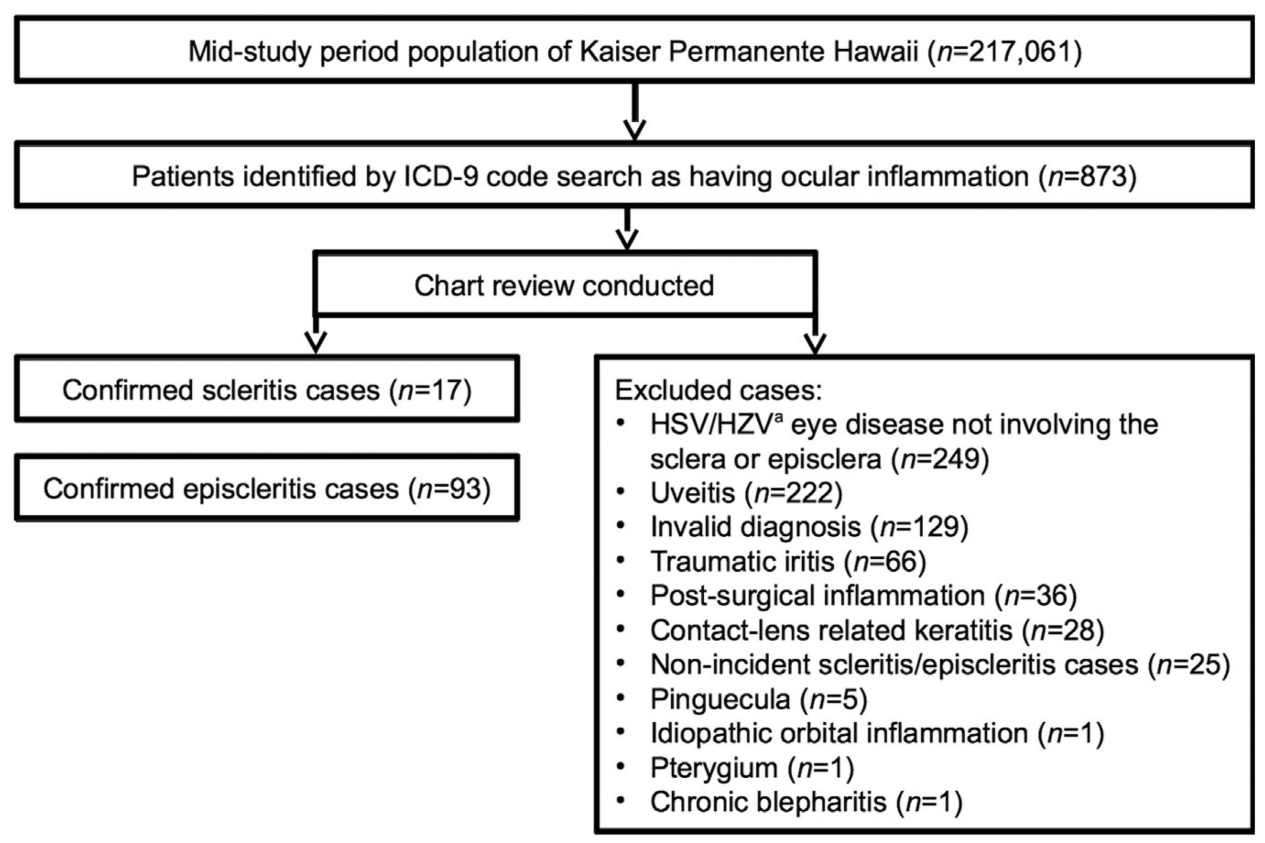

${ }^{\text {a }} \mathrm{HSV}=$ herpes simplex virus, $\mathrm{HZV}=$ herpes zoster virus

Figure.

Study profile for identification of scleritis and episcleritis cases in the Kaiser Permanente Hawaii population. 


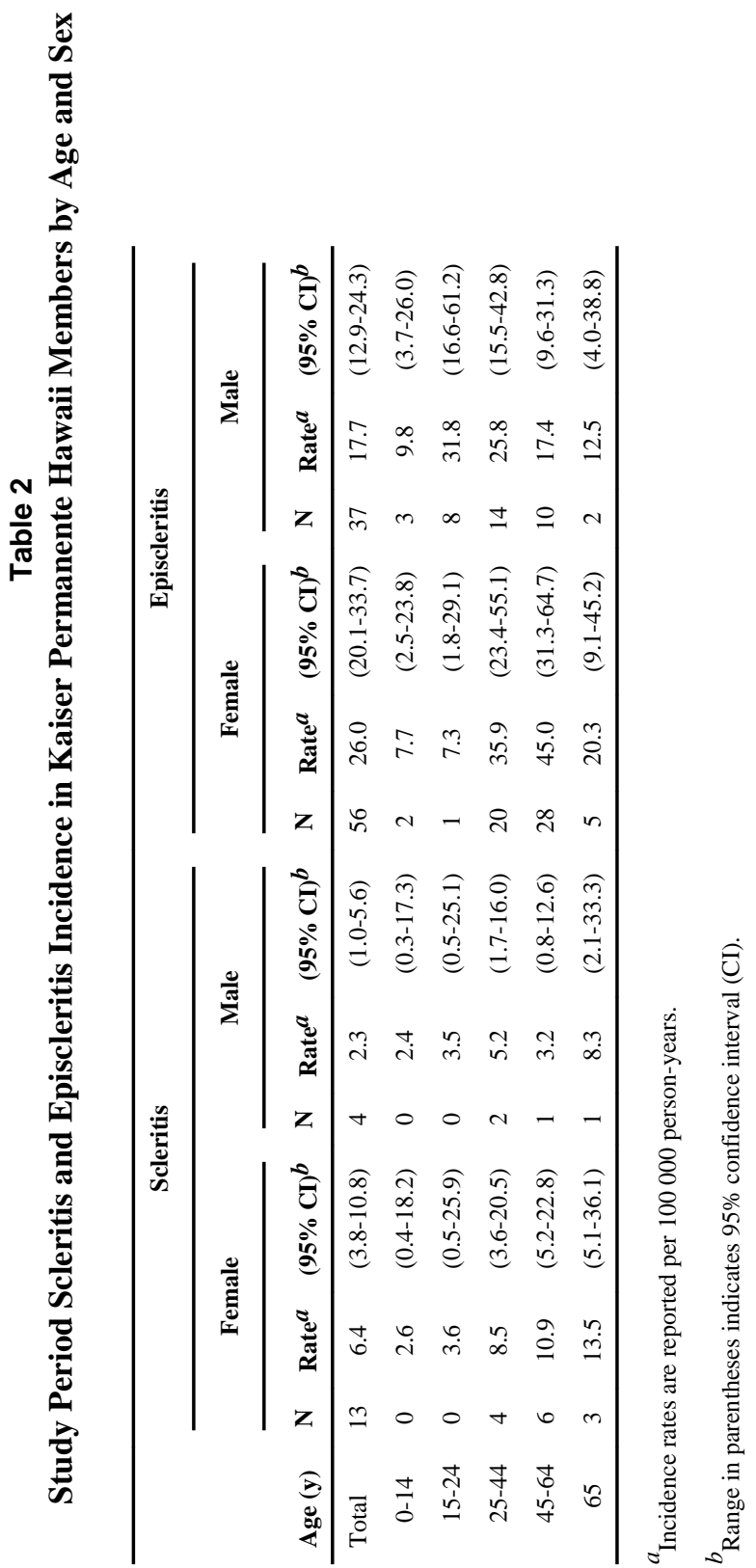


Table 3

Scleritis and Episcleritis Incidence in Kaiser Permanente Hawaii Members by Race

\begin{tabular}{lccccc}
\hline & \multicolumn{2}{c}{ Scleritis Incidence } & & \multicolumn{2}{c}{ Episcleritis Incidence } \\
\cline { 2 - 3 } Race & Rate $^{\boldsymbol{a}}$ & $\mathbf{( 9 5 \% ~ C I ) ~} \boldsymbol{b}$ & & Rate $^{\boldsymbol{a}}$ & $\mathbf{( 9 5 \% ~ C I )} \boldsymbol{b}$ \\
\hline Overall & 4.1 & $(2.6-6.6)$ & & 21.7 & $(17.7-26.5)$ \\
Alaskan/Native American & 33.6 & $(4.7-238.5)$ & & 33.6 & $(4.7-238.5)$ \\
Asian & 10.8 & $(5.8-20.0)$ & & 30.2 & $(20.9-43.7)$ \\
Black & 83.9 & $(27.1-260.2)$ & & 28.0 & $(3.9-198.6)$ \\
Pacific Islander & 1.6 & $(0.2-11.3)$ & 19.1 & $(10.8-33.6)$ \\
White & 9.7 & $(4.4-21.6)$ & 48.6 & $(34.0-69.5)$ \\
\hline
\end{tabular}

$a_{\text {Incidence rates are reported per } 100000 \text { person-years. }}$

${ }^{b}$ Range in parentheses indicates $95 \%$ confidence interval (CI). 